\title{
INTRODUCTIONS D'ÉCREVISSES EN FRANCE ET DANS LE MONDE, HISTORIQUE ET CONSÉQUENCES.
}

\author{
P.J. LAURENT \\ Association des Astaciculteurs de France, Avonnex Marin, 74200 Thonon, France.
}

RÉSUMÉ

Les transferts d'écrevisses se multiplient à la fin du $19^{\text {eme }}$ siècle pour repeupler les innombrables sites dévastés par une maladie nouvelle : la "peste " ramenée d'Amérique du Nord. Si les repeuplements faits avec des sujets autochtones sains se montrent bien décevants, l'introduction en Allemagne de quelques Orconectes limosus de la côte est des États-Unis se révèle si incroyablement efficace qu'aujourd'hui la France et tous les bassins fluviaux d'Europe sont colonisés. Animal facilement migrateur, précoce, fécond, résistant et agressif, la nouvelle écrevisse américaine est aidée dans sa colonisation par une active propagation humaine. O. limosus reste de taille médiocre, ne jouit pas d'une bonne réputation gastronomique, peut propager la "peste " à laquelle il résiste et élimine les espèces autochtones des biotopes dans lesquels il pénètre.

Dans les années 1960, on croit avoir trouvé l'espèce américaine idéale pour remplacer Astacus astacus là où il est décimé par la " peste". On acclimate en Suède Pacifastacus leniusculus, d'une taille très comparable au " pieds rouges", mais il s'avère être aussi un vecteur potentiel de la " peste " et, là où il s'acclimate, il élimine les dernières écrevisses autochtones.

L'écrevisse rouge des marais de Louisiane, Procambarus clarkii, a été jusqu'à maintenant l'écrevisse américaine la plus répandue à travers le monde, exception faite de l'Australie. Une forte production, une croissance rapide, une grande fécondité ont pu séduire ses nombreux propagateurs. Mais ces aspects positifs ne doivent pas cacher sa nature très migratrice, son agressivité vis-à-vis des autres écrevisses, son rôle de vecteur de la " peste " et son incroyable activité fouisseuse. A l'heure actuelle, $P$. clarkii est devenu un fléau dans la plupart des zones où il s'est acclimaté.

La propagation des écrevisses exotiques comporte de grands risques, celle des espèces américaines a été une grave erreur qui a entrainé et entraînera encore des bouleversements préjudiciables à l'environnement aquatique. Quelques mesures sont proposées pour tenter de limiter les effets néfastes des écrevisses américaines sur les peuplements autochtones.

\section{CRAYFISH INTRODUCTIONS INTO FRANCE AND IN THE WORLD, HISTORY AND CONSEQUENCES.}

\section{SUMMARY}

At the end of the 19 th century, crayfish propagation became frequent. At that time, many lakes and rivers have been depopulated by a new illness: " the plague ". The agent of "the plague " has likely been introduced from North America where it exists permanently among native crayfishes. "The plague " does not affect American crayfish but the animal can carry the 
parasite and transmit it to the very susceptible European species. At that time. these facts were ignored, and as restockings made with native species gave poor results, several attempts to introduce American crayfish were made.

The introduction into Germany of some Orconectes limosus, a crayfish from the East coast of USA. gave a so successful result that now not only all France but most of the European rivers are colonized. $O$. limosus migrates easily, is precocious, prolific. withstanding and aggressive. It spreaded not only easily itself but also owing to an active human propagation. The benefit of $O$. himosus settlement in Europe is questionable.

Pacifastacus leniusculus appeared in the 60's as the best substitute of the native Astacus astacus. It has been acclimatized in Sweden. P. leniusculus is a large crayfish very similar to $A$. astacus. But $P$. leniusculus is also a potential vector of "the plague " and eliminates native species.

Until now, red swamp crayfish, Procambarus clarkii, has been the most propagated American species around the world. except Australia. High productivity, rapid growth. great fecundity are the arguments in favour of this species, but it is a very active migrator, an aggressive animal with other crayfishes. a potential vector of "the plague " and especially a very active burrower. Where $P$. clarkii settles, it becomes rapidly a disaster.

Crayfish introduction is always uncertain and risky. American crayfish introductions have been a mistake with bad immediate and future consequences for aquatic environment. Some measures are suggested to reduce the impact of American species.

\section{INTRODUCTION}

Les ecrevisses, décapodes d'eau douce supportant sans peine une longue exposition à l'air. ont offert de très grandes facilités de transplantation.

Dès les temps préhistoriques, les hommes ont vraisemblablement transportè des écrevisses d'un milieu aquatique à un autre (SPITZY, 1979).

Au Moyen Âge, des transplantations d'écrevisses ont été certainement réalisées en Europe.

Mais il faut attendre la seconde moitie du 19... siecle pour que commencent des transferts d'écrevisses d'un continent à l'autre. avec pour consequence le remplacement de populations d'especes autochtones par des espèces exotiques parfois sur de vastes etendues.

Au $20^{\prime \prime}$ siècle. ces transports intercontinentaux se généralisent. engendrant alors des implantations d'écrevisses dans des zones totalement depourvues de ces animaux à l'état naturel. De telles acclimatations conduisent d'une part à la création de ressources aquatiques exploitables nouvelles, mais egalement a des perturbations souvent profondes et dommageables a l'environnement (LAURENT, 1983).

Jusqu'à présent. seul le continent australien a échappe a l'apport d'écrevisses exotiques. et c'est probablement pour cette raison qu'il a conservé une faune riche et originale. Par contre, sur les autres continents livres à la colonisation d'especes nouvelles. on a observé de protonds bouleversements pouvant aller jusqu'à une menace de disparition totale des espèces autochtones. La raison principale est que. pour tous les animaux transplantés et notamment pour les organismes aquatiques. on a introduit avec eux des parasites ou des agents pathogenes nouveaux dont les effets ont pu être désastreux sur les populations en place.

Les ecrevisses, volontiers amphibies et vagabondes. se sont immanquablement échappees des enclos d'élevage malgré les précautions prises pour éviter leur fuite. Eles se sont alors répandues dans la nature migrant naturellement. I'homme a facilité encore leur propagation de façon volontaire ou involontaire, comme par exemple les pêcheurs utilisant des écrevisses comme appáts. ou les aquariophiles. On ne dispose d'aucune donnée sur le rôle 
joué dans les transports d'ecrevisses par certains animaux aquatiques tels que les oiseaux, mais il es: probable qu'ils constituent un facteur supplementaire de dispersion des écrevisses ou de leurs parasites et agents pathogènes.

Globalement. et en depit de quelques aspects positifs, les transferts d'écrevisses exotiques se sont révélés être des erreurs irréversibles qu'il aurait mieux valu ne pas commettre.

\section{LES TRANSFERTS LES PLUS ANCIENS}

It ne s'agissait que de transferts d'animaux provenant de la même région. Ils concernaient des milieux que l'on voulait peupler pour des raisons gastronomiques ou économiques, mais que les écrevisses n'avaient pas pu atteindre de maniere naturelle. par exemple en raison de l'existence de chutes importantes. Ce sont les seigneurs et les moines qui ont réalisé ces actions au Moyen Âge. On en retrouve rarement la trace dans des documents, mais le caractère isolé de certaines populations permet de ne pas douter de tels transferts. On peut citer quelques exemples:

- Dans le canton de Vaud, en Suisse, le lac de Brêt est situé sur le bassin du Léman où Astacus astacus L. n'existe pas à l'état naturel. Ce plan d'eau aurait été peuplé de cette espèce par des Cisterciens de l'abbaye du Haut-Crêt (MURISIER, 1922). Ce peuplement ancien s'est maintenu jusque dans les années 1980 où il a été d'abord concurrencé par un déversement occulte d'Astacus leptodactylus Esch. (tableau 1), puis par celui d'Orconectes limosus (tableau l) Rafinesque, qui a probablement apporté une epidémie fatale à toutes les écrevisses européennes disparues aujourd'hui.

\section{Tableau I}

\section{Écrevisses présentes en France.}

Table I

Crayfishes living in France.

\begin{tabular}{|c|c|c|}
\hline Genre, espèce (origine) & Date d'introduction & Raisons et impact \\
\hline $\begin{array}{l}\text { AUTOCHTONES } \\
\text { Astacus astacus } \mathrm{L} \text {. } \\
\text { Pieds rouges } \\
\text { Austropotamobus pathipes } \\
\text { Lereboullet } \\
\text { Pieds blancs }\end{array}$ & $\ldots$ & \\
\hline $\begin{array}{l}\text { Austropotamobius forrentium } \\
\text { Schrank } \\
\text { Ecrevisse de torent } \\
\text { EXOTIQUES }\end{array}$ & $\ldots \ldots$. & \\
\hline $\begin{array}{l}\text { Orconectes limosus } \\
\text { Rafinesque } \\
\text { Ecrevisse americaine } \\
\text { Côte est des Ėtats-Unis }\end{array}$ & Entre 1911 et 1913 & $\begin{array}{l}\text { Remplacement autochtones } \\
\text { Elimination autochtones }\end{array}$ \\
\hline $\begin{array}{l}\text { Astacus ieptodactylus Esch. } \\
\text { Pattes grèles } \\
\text { Europe de l'es. } \\
\text { Pacrastacus leniusculus } \\
\text { Dana } \\
\text { Ecrevisse de Californie } \\
\text { Cóte ouest dA Amérique du Nord }\end{array}$ & $\begin{array}{l}\text { Fin du } 19 \text { ème } \\
\text { A partır de } 1973\end{array}$ & $\begin{array}{l}\text { Remplacement pieds rouges } \\
\text { apres "peste". } \\
\text { Elimination pieds rouges } \\
\text { Remplacement autochtones } \\
\text { Elımination autochtones }\end{array}$ \\
\hline $\begin{array}{l}\text { Procambarus clarkii Girard } \\
\text { Ecrevisse de Louisiane } \\
\text { Sud-Est des Etats-Unis }\end{array}$ & A partir de 1976 & $\begin{array}{l}\text { Production d'écrevisses } \\
\text { Elimination des autres } \\
\text { écrevisses, dégâts aux } \\
\text { berges }\end{array}$ \\
\hline
\end{tabular}


- La haute vallée de l'Orbe, dans le Jura. au-dessus des pertes infranchissables de cette rivière, aurait également été peuplée d'Austropotamobius pallipes Lereboullet (tableau I) par des moines Bénédictins et Prémontrés (MURISIER 1922).

- En Suisse, dans le canton central dUUri, le lac de Seelisberg n'est relie au système hydrographique que par un émissaire souterrain infranchissable par $A$. astacus. Il était pourtant peuplé de cette écrevisse dont l'introduction, antérieure au $15^{-\cdots}$ siècle, serait due aux moines de l'abbaye de Frauenmünster ou à la seigneurie locale (CARL, 1920).

- De très vastes régions, comme le sud de la péninsule scandinave (Suede, Norvège) ne contiendraient des $A$. astacus qu'à la suite de transferts de ces animaux depuis l'Allemagne, à une époque très ancienne (ABRAHAMSON, 1973).

\section{LES INTRODUCTIONS D'ÉCREVISSES DEPUIS LA SECONDE MOITIÉ DU $19^{\text {eme }}$ SIÈCLE}

La motivation essentielle du début des introductions intercontinentales d'écrevisses a été l'apparition au début des années 1860 en Italie, sur la vallée du Pô dans la province de Trévise, non loin de Venise, d'une épidémie inconnue jusqu'alors parmi les écrevisses d'Europe (ALDERMAN et POLGLASE, 1988). On appela "peste " cette épizootie à propagation très rapide. Elle frappa toute l'Europe à l'exception, à l'époque, des îles britanniques et de la péninsule lbérique. La "peste " sévit encore actuellement de manière sporadique en Europe. L'arrivée de l'épizootie coïncida avec le développement du trafic maritime entre l'Europe et la côte est de l'Amérique du Nord dans les années 1860, epoque du " gold rush ". L'agent pathogène, présent parmi les populations d'écrevisses d'Amérique du Nord, aurait été ramené du Mississippi dans le ballast d'eau douce que les capitaines ne manquaient pas de faire ajouter à leur navire revenant presqu'à vide en Europe (FÜRST, 1982). Ce transfert humain inconscient a eu des conséquences immédiates incalculables dont les effets sont encore d'actualité sur les populations astacologiques de l'Europe.

L'agent de la " peste " n'a été identifie qu'une quarantaine d'années après l'apparition de l'épizootie (SCHIKORA, 1903) et ce n'est qu'au début des années 1930, en reproduisant la maladie sur des sujets sains, qu'on a démontré la nature fongique de la maladie (NYBELIN, 1934). La lenteur et les contradictions des recherches entreprises ont permis une extension générale de la "peste " à pratiquement toute l'Europe.

Le dépeuplement quasi général des eaux à écrevisses engendra un puissant effort de repeuplement soit avec des sujets autochtones réputés indemnes de maladie, soit avec des écrevisses exotiques. En dépit d'un nombre très important de déversements d'écrevisses autochtones, très peu ont abouti à la reconstitution des populations d'origine. Pourtant, peu à peu, les écrevisses européennes sont réapparues dans de nombreux endroits entre les deux guerres. Ces repeuplements se sont faits de manière naturelle à partir de petites colonies d'animaux épargnés par la "peste " et vivant dans des sites isolés par des obstacles tels que des chutes d'eau. On a cependant noté des réintroductions fructueuses : ainsi, De DROUIN de BOUVILLE note l'apparition en 1909 de $A$. pallipes dans un ruisseau de Sologne qui avait été totalement dépeuplé par la "peste " en 1889 des seuls $A$. astacus qu'il contenait. Un déversement avait eu lieu en 1904.

Mais l'idée de remplacer les écrevisses autochtones par une espèce exotique s'est rapidement imposée à l'époque. En France, c'est en 1897 qu'on tente sans succès d'acclimater l'espèce américaine Orconectes virilis Hagen (RAVERET WATTEL, 1897). En Allemagne, c'est VON DEM BORNE, un pisciculteur, qui introduit en 1890 des $O$. limosus dans des étangs du système hydrographique de l'Oder (De DROUIN de BOUVILLE, 1910 ; SCHWENG, 1973). Cette espèce arrive en France un peu plus tard, entre 1911 et 1913, dans la région de Saint-Florent / Cher (BUFFAULT, 1925).

Le bassin de l'Oder en Allemagne d'une part, et celui du Cher en France d'autre part, sont les points de départ de l'écrevisse américaine qui va peu à peu occuper toute l'Europe. 
En France, en 1924, on note l'invasion du Cher (LEGER, 1924) et, dès 1931, les pêcheurs se plaignent de l'abondance des écrevisses (ANDRE, 1960). O. limosus se rencontre dans le bassin de la Seine, en région parisienne, dans les années 1930, puis dans celui de la Saône après la seconde guerre mondiale (LEGER, 1945). Le bassin de la Garonne commence à être colonisé dans les années 1960 (LAURENT et SUSCILLON, 1962). Actuellement, on peut considérer que l'ensemble du territoire français est pratiquement colonisé.

La population allemande du bassin de l'Oder a migré en direction de l'est et s'est retrouvée en Pologne, dont elle occupe une large portion du pays jusqu'aux lacs de Mazurie à l'est (KOSSAKOWSKI, 1973). A l'heure actuelle, elle a poussé sa migration jusqu'en Lituanie (KOREIVA, 1994). A l'ouest de l'Oder, $O$. limosus demeura à l'est de l'Elbe jusque dans les années 1940 (SCHWENG, 1973). En 1955, on la trouve au confluent du Main, où elle est arrivée par les canaux, et du Rhin qu'elle occupe sur une distance de plus de $250 \mathrm{~km}$ en 1972 (SCHWENG, 1973). En 1972, elle est encore inconnue du bassin du Danube mais elle y apparaît au début des années 1990 (TROSCHEL et DEHUS, 1993). Inconnue d'Italie, elle apparaît récemment dans la province de Brescia sur le lac d'Iseo relié au bassin du Pô (DELMASTRO, 1992). Aujourd'hui, seules les îles britanniques, l'Espagne, le Portugal et la Scandinavie ne connaissent pas $O$. limosus.

L'Afrique était le seul continent naturellement dépourvu d'écrevisses mais, dès 1930, des français ont acclimaté $A$. astacus (tableau I) dans des ruisseaux de l'Atlas marocain où il existe encore aujourd'hui. Le pieds rouges a donc été la première espèce présente en terre africaine. Après la seconde guerre mondiale, ce sont des $O$. limosus qui ont été implantés dans de nombreux lacs de l'Atlas où ils ont fait souche.

La propagation de 0 . limosus, devenue commune à l'échelle d'un continent, a-t-elle été bénéfique? Sous l'angle de la pêche, cette espèce est certes capturable, mais si elle intéresse localement quelques pêcheurs amateurs, elle ne fait pas l'objet d'une exploitation commerciale importante. Sa capture reste localisée, en France, à quelques zones où des pêcheurs professionnels, sans réellement la rechercher, en tirent parti. C'est le cas notamment de certaines portions de la Saône ou encore des grands lacs savoyards. Le bénéfice de l'exploitation par ia pêche reste donc très marginal, notamment par le fait que l'espèce jouit d'une réputation gastronomique médiocre. Par contre, les impacts négatifs sur l'environnement astacologique sont réels. D'une part, l'écrevisse américaine demeure toujours un vecteur potentiel de l'agent de la " peste " (VEY et al., 1983); d'autre part, elle a éliminé inexorablement les espèces autochtones avec lesquelles elle a été en contact. Bon nombre de sites potentiellement propices soit à $A$. astacus soit à $A$. leptodactylus, des espèces européennes économiquement beaucoup plus intéressantes, sont irrémédiablement occupés par la petite écrevisse américaine. $O$. limosus est une espèce migratrice active mais l'homme est responsable de la généralisation de sa dispersion. Outre l'ignorance, les repeuplements en poissons provenant d'étangs colonisés d'écrevisses américaines ont été souvent le point de départ de nouvelles colonies de 0 . limosus.

Pacifastacus, un genre d'Astacidae de la côte pacifique d'Amérique du Nord (tableau I), a été introduit au Japon dans les années 1930. On retrouve actuellement Pacifastacus trowbridgii Stimpson au lac Mashu dans l'île septentrionale de Hokkaido, par ailleurs peuplée du seul genre extrême-oriental de Cambaridae, le genre Cambaroides. Au centre de Honshu existe encore, également depuis cette époque, une population de Pacifastacus leniusculus Dana. Ces écrevisses acclimatées au Japon n'ont pas étendu leur territoire car il n'y a eu ni colonisation naturelle ni propagation humaine (LAURENT, 1986).

En Europe, SVÄRDSON en 1960 tente l'acclimatation en Suède de deux espèces américaines: d'une part $P$. leniusculus et d'autre part $O$. virilis. La seconde disparaît mais la première se reproduit et établit une première population européenne. L'objectif de ces premiers essais suédois est de trouver une écrevisse exotique pour remplacer les "pieds rouges" autochtones décimés par la "peste ". Pour éviter une nouvelle transplantation empirique, ABRAHAMSON réalise aux États-Unis et en Suède une étude comparative de l'espèce 
autochtone et de $P$. leniusculus. Les conclusions favorables de cet auteur en ce qui concerne la croissance, la fécondité et la précocité de l'espèce américaine incitent les autorités suédoises à accepter la transplantation d'un lot important de $P$. leniusculus dans les zones méridionales du pays ravagées par la "peste ". Par contre, un programme de restauration des peuplements de " pieds rouges " est entrepris dans la zone nord, isolée et interdite à la propagation de l'espèce américaine. En 1969, 60000 adultes de $P$. leniusculus provenant du lac Tahoe en Californie sont mis en quarantaine, puis répartis dans 69 lacs ravagés antérieurement par la " peste ". Il est décidé peu de temps après de mettre en route une importante écloserie capable de fournir en nombre suffisant les sujets nécessaires pour poursuivre le programme d'acclimatation. Un nouveau lot de 50000 adultes du lac Tahoe constitue le cheptel de base à partir duquel de premiers juvéniles sont obtenus en 1970, puis 200000 en 1971. Cette production se poursuit, devient excédentaire pour la Suède, et l'écloserie tire alors bénéfice de la vente de sujets de repeuplement à toute l'Europe (LOWERY et HOLDICH, 1988). Pratiquement tous les pays européens acclimatent $P$. leniusculus. L'argument justificatif essentiel d'une telle politique est qu'il n'y a pas de risque de contamination des écrevisses autochtones par la nouvelle écrevisse américaine, garantie indemne d'aphanomycose par les vendeurs suédois. Hélas, l'exemple suédois fait école et d'autres écloseries s'établissent, important directement des États-Unis des adultes sanitairement non contrôlés. D'autre part, le commerce des écrevisses de consommation importe également d'Amérique des animaux vivants éventuellement contaminés par l'agent de la "peste " auquel ils résistent très bien dans les conditions habituelles. Pour éviter un tel risque, la Suède a interdit toute importation d'écrevisses vivantes, mais elle a été la seule et la première à le faire à temps. Le défaut de précautions dans les autres pays conduit à une recrudescence de la "peste ". La GrandeBretagne, épargnée à la fin du $19^{\text {eme }}$ siècle, subit à partir de 1981 des hécatombes de sa seule espèce autochtone, le "pieds blancs". Aucune preuve scientifique ne permet de mettre en cause $P$. leniusculus dans la transmission de la maladie, mais il est frappant de constater qu'elle coincide, dans le temps et dans l'espace, avec l'acclimatation de cette écrevisse américaine dans des eaux privées où l'on voulait en faire l'élevage. On reconnaît également que si $P$. leniusculus est pratiquement insensible à la " peste " dans son pays d'origine, placée dans des conditions défavorables en Europe elle peut être mortellement atteinte par le champignon parasite (POLGLASE et ALDERMAN, 1984).

Des " pieds rouges " grecs ont également été les victimes d'aphanomycose à la suite d'essais d'acclimatation de P. leniusculus (THEOCARIS, 1986). L'épidémie turque des années 1980 aurait également pour origine des tentatives d'acclimatation de $P$. leniusculus.

Apporter la preuve scientifique de telles contaminations est toujours très difficile et, d'autre part, les intérêts économiques liés au commerce de $P$. leniusculus tentent d'occulter la vérité. Dans notre pays où les peuplements d'écrevisses autochtones sont devenus dispersés, d'éventuelles attaques de "peste " passent inaperçues, mais certaines mortalités ponctuelles semblent attribuables à cette maladie.

Animal agressif et vigoureux, $P$. leniusculus est également un redoutable concurrent pour les espèces européennes. En cohabitation avec le "pieds rouges", il lui inflige de multiples mutilations (LAURENT et PARIS, 1994). On a également observé que les jeunes " pieds rouges" constituent les proies préférées des petites $P$. leniusculus (KELLER, communication personnelle). On a essayé d'opposer l'agressivité de $P$. leniusculus à celle d'autres écrevisses américaines moins intéressantes, mais ces essais entrepris en Espagne entre $P$. clarkii et $P$. leniusculus ont été peu concluants jusqu'à présent.

Cependant, après l'acclimatation de $P$. leniusculus dans un milieu naturel propice, la pêche de cet animal apprécié constitue une ressource non négligeable. Ainsi au lac Tahoe (Californie), où $P$. leniusculus aurait été apporté par des français émigrés dans la région, on a estimé le stock d'animaux de plus de $20 \mathrm{~g}$ à $1000 \mathrm{t}$ (ABRAHAMSON et GOLDMAN, 1970). Dans le même état, la Sacramento river, où $P$. leniusculus aurait été également acclimaté, la pêche professionnelle très organisée a rapporté jusqu'à 200 t d'écrevisses par an. Dans le lac suédois Hjälmaren, autrefois l'un des plus fameux pour sa pêche aux écrevisses "pieds rouges " 
détruites par la "peste ", $P$. leniusculus a été acclimaté. Treize ans plus tard, la pêche professionnelle capture $25 \mathrm{t}$ d'écrevisses et on estime sa pêche potentielle future à $350 \mathrm{t}$ (ANONYME, 1995 c). La pêche professionnelle du Léman français a produit officiellement une dizaine de tonnes d'écrevisses en 1995 (en majorité $P$. leniusculus), mais ce chiffre peut être facilement doublé si l'on veut cerner la vérité de plus près.

$P$. leniusculus se prête également bien à une production extensive améliorée d'écrevisses en bassin. La Suède et la Grande-Bretagne (HOLDlCH et al., 1995) ont développé de semblables installations. En France, quelques initiatives ont également été prises, parfois avant la publication des mesures législatives interdisant le transport des écrevisses exotiques à l'état vivant. La poursuite plus ou moins occulte de telles activités engendre maintenant des difficultés d'autant plus grandes que, contrairement à la Suède, aucune zonation n'a été déterminée et donc les populations de $P$. leniusculus sont très dispersées.

On croyait l'écrevisse à pattes grêles, $A$. leptodactylus, réfractaire à la " peste " et, lors des grandes épidémies d'aphanomycose, on l'a introduite dans un grand nombre de plans d'eau où les "pieds rouges " avaient été décimés. Là où la "peste " n’a plus sévi, ces écrevisses plus prolifiques, plus précoces et à croissance rapide ont donné des populations qui ont proliféré, ont migré et se sont retrouvées en cohabitation avec $A$. astacus. Ces contacts ont été fatals aux « pieds rouges " finalement supplantés par l'écrevisse à pattes grêles (CUKERZIS, 1970). A. leptodactylus peut générer des productions intéressantes dans les milieux trop chauds défavorables aux $A$. astacus. La chute de la production turque à la suite des attaques d'aphanomycose des années 1980 ouvre de bonnes perspectives commerciales aux élevages extensifs dont on commence à voir un début d'organisation.

Les japonais pratiquent l'élevage de la grenouille boeuf Rana catesbeiana depuis 1918 et sont à la recherche d'aliments vivants. Ils songent à Procambarus clarkii Girard (tableau I), une écrevisse des marécages de Louisiane. En 1930, ils font revenir 100 sujets de la NouvelleOrléans (PENN, 1954). A l'arrivée au Japon, il ne reste que 20 individus vivants. Ils prennent place dans l'élevage, se multiplient, s'échappent, envahissent les rizières voisines de Kwanto et d'Osaka, ravageant les récoltes et minant les digues par leur activité fouisseuse. Originaires de régions à fortes variations de régime hydrologique, ces animaux, comme beaucoup d'autres écrevisses, ont développé l'enfouissement profond comme moyen de défense contre les sécheresses prolongées. L'invasion du Japon devient générale, à l'exception de l'île septentrionale d'Hokkaido ; les aquariophiles sont en partie responsables de la rapidité et du succès de la dispersion de l'espèce.

A partir de 1948, P. clarkii devient une calamité agricole au Japon. On peut compter jusqu'à 100000 individus par hectare de rizière et estimer la biomasse présente à $1200 \mathrm{~kg}$ par hectare (LAURENT, 1986). Les moyens de destruction mis en oeuvre (produits chimiques, action du froid hivernal) s'avèrent inefficaces (KAMITA, 1970). De nos jours, l'explosion de population est terminée et, plus de 60 ans après leur introduction, les écrevisses ont perdu leur ardeur colonisatrice. Les japonais attribuent ce changement aux effets répétés des pesticides à usage agricole et à la pollution des eaux. Le Japon a été la première étape asiatique de $P$. clarkii.

Durant la guerre, des soldats japonais apportèrent des $P$. clarkii dans le lac d'un jardin public de Nankin. L'animal s'adapte à ces nouvelles conditions, se multiplie discrètement et soudain se signale par les dégâts qu'il commet aux systèmes d'irrigation. Actuellement, $P$. clarkii occupe les territoires de 13 provinces chinoises (XINYA, 1988). D'abord considérée comme ennemi agricole et non consommée par les autochtones, l'écrevisse rouge de Louisiane devient l'objet de pêches intensives pour alimenter les marchés locaux conquis par ce nouvel aliment et surtout pour l'exportation. Selon une estimation de 1992 (ANONYME, 1995 a), la production chinoise annuelle aurait atteint $40000 \mathrm{t}$, soit un peu moins que la quantité maximum récoltée aux États-Unis. Aujourd'hui, la Chine exporte à des conditions défiant toute concurrence et vend même des écrevisses aux États-Unis, pourtant premier producteur mondial. 
En Afrique, on a cru trouver en $P$. clarkii un moyen de lutte biologique contre les mollusques aquatiques vecteurs de la bilharziose et apporter par cet animal des protéines à des populations sous-alimentées. L'Ouganda et le Kenya sont ensemencés entre 1963 et 1970. C'est de ces endroits que sont parties les nombreuses populations établies maintenant en Afrique. Le peuplement du lac Naivasha au Kenya est un succès qui dépasse toutes les espérances. On estime que la capture quotidienne de $1 \mathrm{t}$ d'écrevisses pendant 300 jours par an serait incapable d'épuiser le stock (MIKKOLA, 1978). Cette énorme production est toutefois peu exploitée par absence de consommation locale et difficultés logistiques d'organisation des exportations. Seules pour l'instant en Afrique les $P$. clarkii de Zambie semblent générer des profits (GRUBB, 1982). L'Afrique du Sud, le Soudan, l'Egypte, et peut-être d'autres contrées africaines possèdent désormais des écrevisses dont la pullulation est à redouter si une exploitation intensive ne se développe pas.

P. clarkii arrive en Europe par l'Espagne à partir de 1973. Les performances de la production en Louisiane (jusqu'à près de $4 \mathrm{t}$ à l'ha) motivent les promoteurs de $P$. clarkii en Espagne. Le premier contingent de 250 mâles et de 240 femelles est placé dans un système de bassins sans écoulement, alimenté en eau par le pompage d'un puits. Des barrières théoriquement infranchissables entourent les installations. Des fuites se produisent cependant et les écrevisses ont accès au bassin du Guadiana, qui leur permettra de gagner le Portugal. En 1974, une autorisation officielle d'acclimatation permet l'importation de $500 \mathrm{~kg}$ d'écrevisses que l'on déverse dans d'anciens bassins de stockage d'anguilles, le long du Guadalquivir. En dépit d'une mortalité de $80 \%$ des animaux importés, les survivants se multiplient rapidement et migrent. Dès 1975, on capture 480 sujets, puis 1,8 t en 1976 et dix fois plus en 1977 (HABSBURGO LORENA, 1978). La colonisation du pays devient rapidement générale, le « pieds blancs " autochtone est éliminé par concurrence directe ou par des attaques répétées d'aphanomycose. Les dégâts aux systèmes sophistiqués d'irrigation sont constants et considérables, mais par ailleurs la pêche fait vivre de pauvres gens et rapporte jusqu'à $3000 \mathrm{t}$. Elle fluctue ensuite énormément, en raison des épisodes de sécheresses sévères que connaît l'Espagne.

Les exportations espagnoles d'animaux vivants, totalement légales en France jusqu'en 1983, fournissent aux " amateurs " les animaux nécessaires à de multiples implantations, en particulier dans le sud du pays (Gers et Gard). Les importateurs pratiquant le retrempage sont également responsables de l'invasion de régions telles que la Brière, à partir d'installations situées à Saint-Lyphar, ou la Charente depuis la région de Brizambourg. On connaît aujourd'hui des colonies isolées, en Angleterre $(\mathrm{HOLDICH}, 1994)$, en Allemagne (TROSCHEL et DEHUS, 1993), en Italie (DELMASTRO, 1994).

Les dégâts aux berges et aux digues constituent les méfaits habituels de $P$. clarkii, mais en Aquitaine on a également commencé à noter d'importantes réductions de production piscicole dans les étangs occupés par cette écrevisse (ROQUEPLO et al., 1995). Sur le plan astacicole, la concurrence exercée sur les espèces autochtones et surtout le risque constant de transmission de l'aphanomycose sont des facteurs décisifs de l'anéantissement des populations locales. En l'absence d'une exploitation intensive organisée, $P$. clarkii ne laisse lors de son acclimatation qu'une série de graves atteintes à l'environnement aquatique et la propagation de cette espèce à l'échelle mondiale a constitué une énorme erreur écologique.

II existe aux États-Unis un type particulier d'astaciculture qui consiste à produire de jeunes écrevisses pour les pêcheurs à la ligne. Dans l'état de New York, cette activité ne concerne pas moins de 109 ha de plans d'eau et il se produit chaque année 27 tonnes d'appâts pour la pêche (ANONYME, 1995 b). Cette activité ne concerne que quelques espèces d'écrevisses, d'une part le "paper shell crawfish": Orconectes immunis Hagen, à la carapace assez fine pour être enfilée sans peine sur les hameçons, et d'autre part Orconectes rusticus Girard, vendue quand elle est molle après la mue. En utilisant cette dernière espèce, les pêcheurs l'ont largement propagée en dehors de son aire de répartition. Au Wisconsin notamment, $O$. rusticus s'est multipliée, a éliminé les autres espèces d'écrevisses, a été accusée de détruire le frai des poissons et surtout a totalement faucardé les herbiers. De tels 
dégâts ont incité à envisager des éradications chimiques de cette espèce (MOMOT, 1988). Les pêcheurs sportifs européens utilisent également des écrevisses pour capturer des Percidés ou le brochet et ils sont souvent à l'origine de peuplements indésirables d'écrevisses, notamment de O. limosus.

L'Australie est très soucieuse de la protection de sa faune et de sa flore et sa vigilance lui a permis d'échapper, jusqu'à présent, à une invasion de ses eaux par une espèce exotique d'écrevisse. Elle n'a ainsi jamais connu d'épidémie de "peste ", maladie à laquelle ses écrevisses sont pourtant aussi sensibles que les espèces européennes. Si l'Australie s'est abstenue d'importer des écrevisses étrangères, elle a largement approvisionné d'autres pays en écrevisses australiennes. Ce sont trois espèces du genre Cherax, d'ailleurs déjà exportées à l'état adulte pour la consommation, qui ont fait l'objet d'un tel commerce. Cherax tenuimanus Smith ou "Marron" de la partie occidentale de l'Australie, Cherax quadricarinatus von Martens ou "Red Claw" de la région septentrionale chaude, tous deux de grande taille, et Cherax destructor Clark ou " Yabby", de taille plus modeste et de la zone sud orientale, sont les trois écrevisses transplantées actuellement dans de nombreux pays. Ces transplantations n'ont pas pour objectif l'acclimatation de ces animaux dans la nature mais la production, si possible intensive, d'écrevisses de consommation. Dans des installations sophistiquées, les rendements annuels en bassins peuvent atteindre 3 à $5 \mathrm{t}$ à l'ha (ACKEFORS, 1994). II n'a pas encore été rapporté d'acclimatation de ces animaux dans la nature d'un des pays importateurs. Le "Yabby ", cependant, espèce très prolifique, très rustique et très fouisseuse, pourrait se comporter de la même manière que $P$. clarkii ; les États-Unis et la Zambie tentent son astaciculture. Le "Marron " fait l'objet de premiers essais au Chili. Le "Red Claw ", espèce franchement tropicale, dont la production intensive semble bien maîtrisée, existe en Israël, au Belize, en Afrique du Sud, en Équateur. Dans ce dernier pays, des sujets de 15 mois peuvent atteindre $290 \mathrm{~g}$. La Chine et l'Espagne expérimentent des productions intensives de Cherax ; la production espagnole, très confidentielle, atteindrait déjà 2 t par an.

\section{MESURES PROPOSÉES POUR TENTER DE LIMITER LES EFFETS NÉFASTES DES ÉCREVISSES AMÉRICAINES SUR LES PEUPLEMENTS AUTOCHTONES}

Les mobiles des transplantations d'écrevisses sont très divers :

- repeuplement de sites dont les populations autochtones ont disparu à la suite d'épizooties ;

- production extensive ou intensive, en milieux théoriquement isolés, de sujets destinés à la consommation humaine.

Beaucoup de transplantations n'ont eu aucun mobile et sont dues au hasard ou à des initiatives non raisonnées.

L'acclimatation d'une espèce exotique à forte résilience conduit à des situations totalement imprévisibles et globalement préjudiciables à l'environnement.

Une telle situation s'aggrave singulièrement lorsqu'il n'y a pas d'exploitation intensive organisée de la nouvelle espèce. Seuls des pêcheurs professionnels, artisans travaillant pour leur compte ou salariés de Services chargés de la gestion de l'environnement, sont capables d'assurer l'exploitation assidue et efficace nécessaire au contrôle des populations. L'exploitation des écrevisses exotiques devrait pouvoir être envisagée, mais leur mise en vente ne se faisant alors que sous forme cuite ou congelée.

Les importations d'écrevisses vivantes sont la source de toute acclimatation indésirable et constituent une menace sanitaire constante pour les populations d'écrevisses autochtones.

Une surveillance permanente de l'état sanitaire des populations d'écrevisses américaines permettrait de détecter les souches infestées d'aphanomycose et de prendre à temps les dispositions nécessaires pour empêcher l'extension de la maladie. 
L'interdiction de transporter vivantes des écrevisses exotiques doit être impérativement maintenue et surtout bien respectée.

Il faudrait mettre fin à l'utilisation d'écrevisses en aquariologie ou comme leurre pour la pêche, activités qui sont souvent le point de départ de peuplements indésirables.

\section{CONCLUSION}

L'impact des espèces exotiques acclimatées s'exerce sur l'ensemble du milieu aquatique. Les berges, les digues et d'une manière générale le réseau hydraulique peuvent être gravement endommagés, les espèces autochtones d'écrevisses sont menacées de disparition totale, le rendement en poissons des plans d'eau semble pouvoir être affecté, bien que la question mérite davantage d'observations. Les plantes aquatiques peuvent être détruites. La pêche cependant peut constituer une ressource nouvelle mais malheureusement la rentabilité économique, dans le cas d'animaux de petite taille et de piètre réputation gastronomique, risque d'être discutable dans un contexte mondial de surproduction. La présence d'écrevisses en abondance peut également affecter favorablement certains vertébrés liés au milieu aquatique : oiseaux ou loutres (DELIBES et ADRIAN, 1987).

L'acclimatation d'une espèce exotique d'écrevisse est un phénomène irréversible, la mise au point d'un astacicide spécifique et sans danger pour l'environnement aquatique reste hautement hypothétique (LAURENT, 1995).

Un siècle après l'apparition des premières écrevisses américaines en Europe, il faudra apprendre à gérer convenablement les populations d'écrevisses exotiques pour tenter de limiter leur pullulation et ralentir leur extension. Le maintien des derniers sites contenant des écrevisses autochtones dépend de notre capacité immédiate à mener à bien les interventions indispensables.

\section{BIBLIOGRAPHIE}

ABRAHAMSON S., 1973. The crayfish Astacus astacus in Sweden and the introduction of the American crayfish Pacifastacus leniusculus. Fresh. Cray., 1, 37-40.

ABRAHAMSON S., GOLDMAN C., 1970. Distribution, density and production of the crayfish Pacifastacus leniusculus (Dana) in lake Tahoe. Oikos, 21, 83-91.

ACKEFORS H., 1994. Recent progress in Australian crayfish culture. World Aquaculture, 25 (4), 14-19.

ALDERMAN D.J., POLGLASE J.L., 1988. Pathogens, parasites and commensals, in HOLDICH D. and LOWERY R. Eds., Freshwater Crayfish, biology, management and exploitation. Croom Helm, London, 498 p.

ANDRE M., 1960. Les écrevisses françaises. Editions Lechevallier, $291 \mathrm{p}$.

ANONYME, 1995 a. News from the Peoples Republic of China. Crayfish News, I.A.A. News Letter, 17 (2), 4.

ANONYME, 1995 b. Commercial crayfish culture in New York USA. Crayfish News, I.A.A. News Letter, 17 (3), 7.

ANONYME, 1995 C. Crayfish news from Sweden. Crayfish News, I.A.A. News Letter, 17 (3), 8.

BUFFAULT P., 1925. Une écrevisse américaine acclimatée en France : le Cambarus du Cher. Rev. des Eaux et Forêts, 63, 274.

CARL J., 1920. Catalogue des invertébrés de la Suisse, fascicule 12, Décapodes. Georg Ed., Genève, $35 \mathrm{p}$. 
CUKERZIS J., 1970. La biologie de l'écrevisse (Astacus astacus L.). Traduction française de ZUZINE N., INRA Publications, Versailles, $313 \mathrm{p}$.

De DROUIN de BOUVILLE R., 1910. La pratique des repeuplements en écrevisses. Bull. de la Sté. Centrale d'Aquiculture et de Pêche, 22, 17-32.

DELIBES M., ADRIAN I., 1987. Effects of crayfish introduction on otter Lutra lutra in the Donana National Park, S.W. Spain. Biol. Conserv., 42, 153-159.

DELMASTRO G.B., 1992. II gambero americano Orconectes limosus (Rafinesque) un nuovo decapodo neoartico nelle acque dolci del nord Italia (Crustacea Decapoda Cambaridae). Natura Breciana, Ann. Mus. Civ. Sc. Nat. Breschia, 27, 171-174.

DELMASTRO G.B., 1994. II gambero della Louisiana un nuovo abitatore delle nostre acque. II Notiziario, Pro Natura Carmagnola, 19.

FÜRST M., 1982. Introduction of the north American crayfish Pacifastacus leniusculus (Dana) into Sweden. F.A.O., Document Technique de la C.E.C.P.I., 42 (2), 400-404.

GRUBB C.J., 1982. Kenya's pest, Zambia's prize. Fish Farming International, 9 (7), 10.

HABSBURGO LORENA A., 1978. Present situation of exotic species of crayfish introduced into Spanish continental waters. Fresh. Cray., 4, 175-184.

HOLDICH D., 1994. A bit of English crayfish humour. Crayfish News, I.A.A. News Letter, 16 (2), 6.

HOLDICH D., REEVE I., ROGERS D., 1995. Introduction and spread of alien crayfish in British waters - implications for native crayfish populations. Fresh. Cray., 8, 99-112.

KAMITA T., 1970. Studies on the freshwater shrimps, prawns and crayfishes of Japon. Nishi-Kawarzu-machi, Matsue, Japon, 159-170 (en japonais, avec résumé anglais).

KOREIVA C., 1994. O. limosus in Lithuania. Crayfish News, I.A.A. News Letter, 16 (3), 7.

KOSSAKOWSKI J., 1973. The freshwater crayfish in Poland. Fresh. Cray., 1, 18-26.

LAURENT P.J., 1983. Un siècle de transplantations d'écrevisses nord-américaines. C.R. Soc. Biogéogr., 59 (3c), 393-404.

LAURENT P.J., 1986. Les écrevisses au Japon. L'Astaciculteur de France, 7, 20-21.

LAURENT P.J., 1995. Eradication of unwanted crayfish species for astacological management purposes. Fresh. Cray., 8, 121-133.

LAURENT P.J., SUSCILLON M., 1962. Les écrevisses en France. Ann. de la Sta. Centr. d'Hydrobiologie Appliquée, 9, 335-395.

LAURENT P.J., PARIS L., 1994. Les mésaventures d'une population de pieds rouges en Morvan. L'Astaciculteur de France, 41, 6-8.

LEGER L., 1924. Une nouvelle écrevisse dans les eaux françaises. C.R. Acad. Sciences, 179, 1205.

LEGER L., 1945. Sur la présence en nombre de l'écrevisse américaine Cambarus dans la basse Saône. C.R. hebd. Séances Acad. Sci. Paris, 221, 14-18.

LOWERY R., HOLDICH D., 1988. Pacifastacus leniusculus in North America and Europe, with details on the distribution of introduced and native craytish species in Europe. In HOLDICH D. and LOWERY R. Eds., Freshwater Crayfish, biology, management and exploitation. Croom Helm, London, 498 p.

MIKKOLA H., 1978. Ecological and social problems in the use of the crayfish Procambarus clarkii in Kenya. Fresh. Cray., 4, 197-205.

MOMOT W., 1988. Orconectes in North America. In HOLDICH D. and LOWERY R. Eds., Freshwater Crayfish, biology, management and exploitation. Croom Helm, London, $498 p$. 
MURISIER P., 1922. Les écrevisses dans les eaux vaudoises en 1917. Bull. de la Sté. Vaudoise de Sci. Nat., 54, 183-192.

NYBELIN O., 1934. Nya undersokingor over Kraftpesten orsak. Ny Svensk Fiskeritidskr., 110-114.

PENN G., 1954. Introductions of American crayfishes into foreign lands. Ecology, 35, 296.

POLGLASE J., ALDERMAN D., 1984. Crayfish plague threatens U.K. stock. Fish Farmer, 7 (3), 16-17.

RAVERET WATTEL M.C., 1897. Le Cambarus virilis à la station aquicole de Fécamps. Bull. de la Sté. Centrale d'Aquiculture et de Pêche, 9, 113.

ROQUEPLO C., LAURENT P.J., NEVEU A., 1995. Procambarus clarkii Girard (écrevisse rouge des marais de Louisiane), synthèse sur les problèmes posés par cette espèce et sur les essais pour contrôler ses populations. L'Astaciculteur de France, 44, 2-14 et 45, 2-17.

SCHIKORA F., 1903. Über die Krebspest und ihre Erreger. Fisch. Zeit., 6, 353-355.

SCHWENG E., 1973. Orconectes limosus in Deutschland insbesondere im Rheingebiet. Fresh. Cray., 1, 79-87.

SPITZY R., 1979. The prehistoric man as a possible crayfish transporter. Fresh. Cray., 4, 221-225.

THEOCARIS U., 1986. La pêche aux écrevisses dans la région d'Hipiros en Grèce. L'Astaciculteur de France, 8, 4-10.

TROSCHEL J., DEHUS P., 1993. Distribution of the crayfish species in the Federal Republic of Germany, with special reference to Austropotamobius pallipes. Fresh. Cray., 9, 390-398.

VEY A., SÖDERHÄLL K., AXAJON R., 1983. Susceptibility of Orconectes limosus Raf. to the crayfish plague Aphanomyces astaci Schikora. Fresh. Cray., 5, 284-291.

XINYA S., 1988. Crayfish and its cultivation in China. Fresh. Cray., 7, 391-395. 